\title{
Unusual Presentation of Spontaneous Coronary Artery Dissection in an Older Male
}

\author{
Rajveer Joea ${ }^{a}$, Sarah J. Strube ${ }^{a}$, b, Todd K. Zynda ${ }^{c, d}$
}

\begin{abstract}
Spontaneous coronary artery dissection (SCAD) is a variant of acute coronary syndrome (ACS) that is poorly understood. SCAD has been linked to fibromuscular dysplasia (FMD), connective tissue disease (CTD), pregnancy and hormonal imbalance, systemic inflammatory conditions (e.g. IBD, vasculitis), and coronary artery vasospasm rather than traditional cardiac risk factors. Symptomology generally accompanying SCAD is indistinguishable from ACS making the timely recognition and diagnosis vital for prompt treatment. Management of SCAD is not well defined given the absence of guidelines; conservative therapy with or without invasive intervention is assessed on a case-by-case basis. In this article, we report the case of a 62-year-old male, who presented with chest pain and dyspnea on exertion and was found to have an elevated troponin-I level and corresponding electrocardiogram (EKG) findings, subsequently diagnosed with a non-ST elevation myocardial infarction (NSTEMI). Coronary angiography revealed a distal right coronary artery (RCA) dissection, which was confirmed later with intravascular ultrasound (IVUS). The patient then underwent percutaneous coronary intervention (PCI) followed by stenting of the distal RCA and was discharged on optimal medical therapy. Herein, we report a case of SCAD in an otherwise healthy male with chest pain at rest and with mild exertion without conventional cardiac risk factors.
\end{abstract}

Keywords: Spontaneous coronary artery dissection; Intravascular ultrasound; Echocardiogram; NSTEMI

\section{Introduction}

Spontaneous coronary artery dissection (SCAD) is a rare cause

Manuscript submitted March 29, 2018, accepted May 4, 2018

${ }^{a}$ Department of Internal Medicine, St Mary Medical Center, Long Beach, CA 90813, USA

bHealth Science, St Mary Medical Center, Long Beach, CA 90813, USA

'Department of Cardiology, St Mary Medical Center, Long Beach, CA 90813, USA

${ }^{\mathrm{d} C}$ Corresponding Author: Todd K. Zynda, St Mary Medical Center, 1045 Atlantic Ave., Suite 611, Long Beach, CA 90813, USA.

Email: zynda06@yahoo.com

doi: https://doi.org/10.14740/cr717w of acute coronary syndrome (ACS), occurring in 0.1 to $0.4 \%$ of cases; characteristically female predominant [1]. SCAD is a phenomenon that involves spontaneous dissection of the coronary intima or media whilst creating both a false lumen and intramural hematoma. Additionally, enlargement of the false lumen secondarily to an expanding hematoma will ultimately cause myocardial ischemia and infarction. SCAD however does not incorporate dissections resulting from trauma or plaque rupture secondary to coronary atherosclerosis and generally is not linked with the well-known risk factors for ACS, although patients do present similarly $[2,3]$. Classically, SCAD is diagnosed with coronary angiography or IVUS and treated medically, followed by percutaneous coronary intervention $(\mathrm{PCI})$, or coronary artery bypass grafting $(\mathrm{CABG})$ to achieve Thrombolysis in Myocardial Infarction (TIMI) 3 flow. The degree of reperfusion is categorized using the TIMI flow grading system with grade 3 flow indicating adequate coronary flow and 0 signifying no perfusion [4].

\section{Case Report}

A 62-year-old male presented with chest pain that started earlier in the day, while delivering mail, recurring for several months. He complained of occasionally experiencing blurry vision, but both review of systems and cardiovascular physical examination were unrevealing. Initial EKG demonstrated less than $1 \mathrm{~mm}$ up-sloping ST depression in leads III and aVF without ST elevation or T wave inversion in other leads. Troponin I was elevated to 0.151 , which peaked at $0.1816 \mathrm{~h}$ later. Transthoracic echocardiogram revealed mild concentric left ventricular hypertrophy with left inferior and posterior ventricular wall hypokinesis and preserved ejection fraction of 55 to $60 \%$. The patient was found to have no other cardiac risk factors other than age that predisposed him to an acute coronary syndrome; urine toxicology screening was negative. Consequently, the patient was diagnosed as an NSTEMI and taken for further evaluation. Cardiac catheterization revealed a small caliber, mid and distally, LAD without focal stenosis, and an obtuse marginal two (OM2) branch, which was small caliber with an $80 \%$ focal hazy lesion and TIMI 3 flow. The RCA was enlarged and ectatic with a linear lucency distally concerning for possible dissection and/or thrombus formation with TIMI 3 flow (Fig. 1, 2). Further investigation with IVUS revealed a false lumen with a prominent dissection in the distal RCA causing a $70 \%$ dynamic stenosis (Fig. 3 ). IVUS confirmed the 


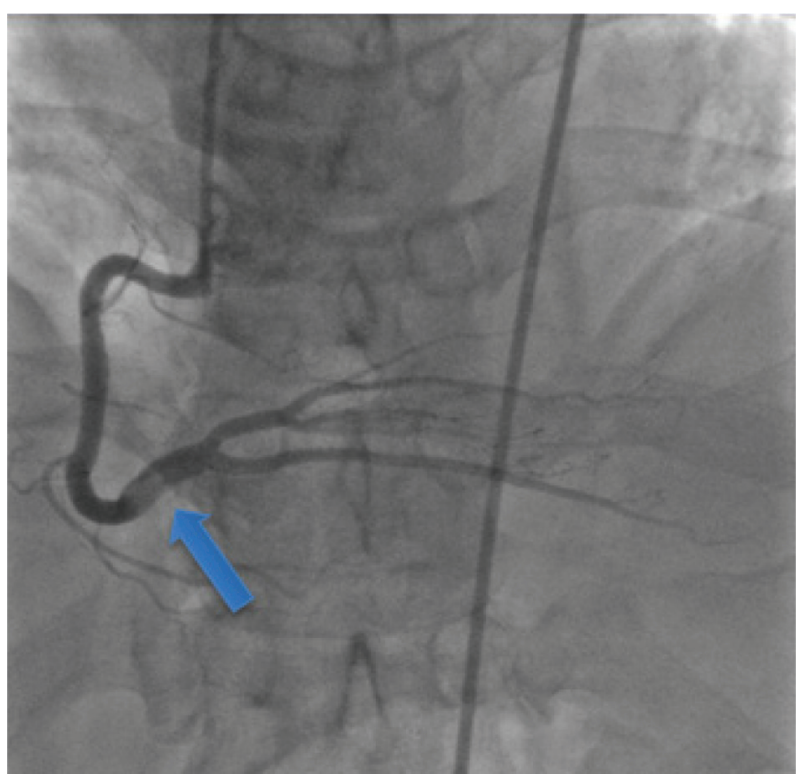

Figure 1. Coronary angiogram reveals a filling defect involving the distal RCA.

distal RCA to be a large and ectatic vessel with a diameter ranging from 5.4 to $6 \mathrm{~mm}$ in the distal portion and 4.5 to 5 $\mathrm{mm}$ in the more proximal portion of the vessel. Sequentially an everolimus-eluting stent $(4 \mathrm{~mm} \times 32 \mathrm{~mm})$ was successfully deployed to the distal RCA and post-dilated under IVUS guidance to a maximum of $5.28 \mathrm{~mm}$ using a $4.5 \mathrm{~mm}$ non-compliant balloon (Fig. 4). The remainder of the hospital stay was uneventful and the patient was subsequently discharged home the following day on dual antiplatelet therapy with aspirin and ticagrelor.

\section{Discussion}

Coronary arteries are composed of three layers: tunica adventitia, media, and intima. The outermost layer or adventitia is comprised of connective tissue primarily collagen fibers, while the media is equal parts smooth muscle and connective tissue chiefly elastic fibers, and the innermost layer or intima is principally endothelial cells [5]. Embedded within these layers is a matrix of small blood vessels recognized as the vasa vasorum. The leading mechanism of SCAD involves injury to the vasa vasorum resulting in the formation of a false lumen with consequent accumulation of blood within the tunica media [6,7]. Concomitantly the false lumen continues to expand by means of pressure gradients, which in turn leads to diminished coronary flow within the true lumen, impaired myocardial perfusion, and imminent myocardial infarction [6].

Recognized precipitants to SCAD include: FMD, CTD, systemic inflammatory conditions (e.g. IBD, vasculitis), coronary artery vasospasm, precipitating stress events (e.g. intense exercise or emotion), recreational drugs and labor and delivery $[1,8]$. Moreover, vasculitic disorders cause inflammation of blood vessels, whereas CTD promotes deterioration of the elastic fiber architecture of the arterial wall. Other stressors

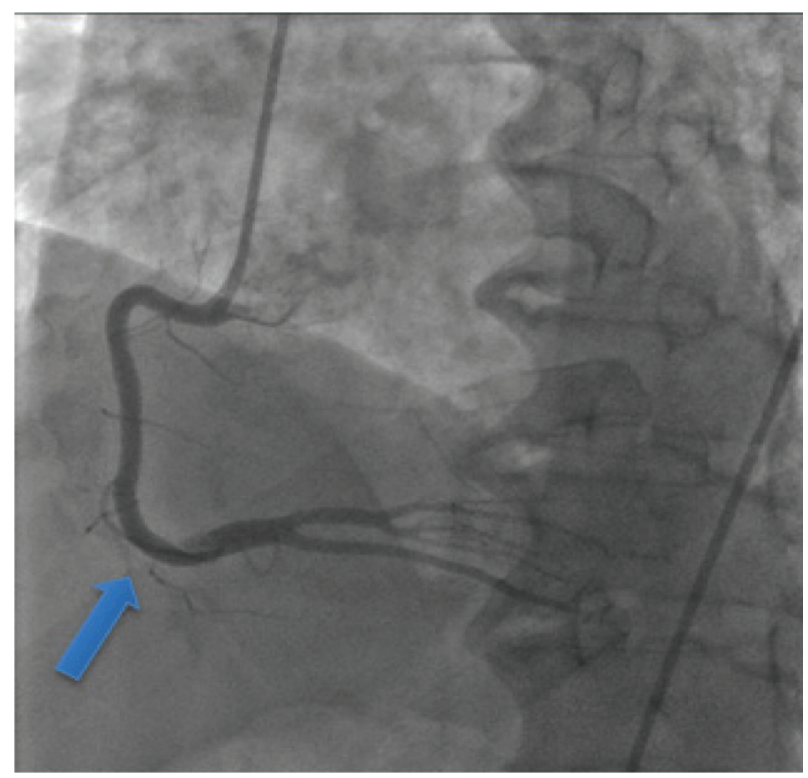

Figure 2. Linear lucency within the distal RCA, suspicious for dissection.

including labor and delivery, stimulant drug use, and intense exercise all augment release of catecholamines generating increased coronary artery sheer stress, i.e., $\mathrm{dP} / \mathrm{dT}[1,5]$. SCAD often involves the left anterior descending (LAD) artery, in the mid to distal portions of vessels, and generally involves only one coronary artery $[1,9]$.

Multiparity and hormone disproportion are other associated links to SCAD. Both estrogen and progesterone are the predominant hormones involved; yet, the added risk associated with SCAD does not depend on the absolute levels, but fluctuations in the concentration of these hormones. Variations in hormone levels are highest throughout pregnancy including the prepartum and postpartum periods and those receiving hormone therapy including birth control, hormone replacement (e.g. testosterone), and infertility treatment. Estrogen may mediate its' effects via an upsurge in shear force, in addition to causing an inflammatory cell infiltration into the arterial wall [10]. Excess progesterone during pregnancy provokes loss of elastic fiber architecture and degeneration of medial wall collagen, resulting in weakening of the arterial wall and subsequent arterial dissection $[8,10,11]$. SCAD development is strongly influenced by chronic hormonal dysregulation, whether exogenous or as a result of frequent pregnancies.

SCAD, recognized as a life-threatening cause of ACS, is uncommon, and its' etiology is poorly understood. SCAD classically affects younger women under the age of 50 in over $80 \%$ of cases; however men and older women remain susceptible, albeit to a lesser degree [12]. SCAD is typically due to non-atherosclerotic etiology, and typical risk factors for heart disease including hypertension, hyperlipidemia, diabetes mellitus, smoking, obesity, and family history of premature coronary artery disease (CAD) may be lacking. The signs and symptoms, cardiac enzymes, and EKG often show changes that parallel ACS. SCAD is ultimately detected by coronary angiography and advanced intracoronary imaging tools (e.g. 


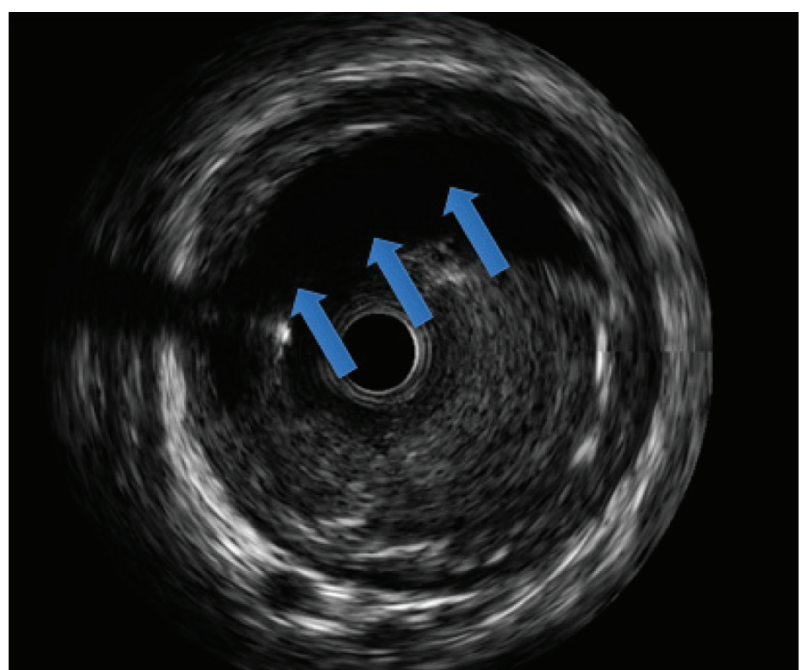

Figure 3. Intravascular ultrasound of an intramural hematoma revealing a false lumen and noticeable dissection of the distal right coronary artery.

optical coherence tomography (OCT)). Sophisticated intracoronary imaging such as IVUS or multi-detector CT can be applied to display intramural hematoma flow or track the dissection over time, respectively. Yet, the majority of cases are not identified given that the intramural hematoma must be of considerable size to cause obstruction of the coronary lumen [11].

Interestingly, our patient was found to have an RCA that was both enlarged and ectatic. Ectasia is dilation or expansion of a tubular structure frequently pathologic $[10,13,14]$. Coronary artery ectasia is distension of an arterial segment, in reference to that of an adjacent normal coronary artery, to a diameter of 1.5 times greater. The Law of Laplace states wall stress is proportional to the intra-arterial pressure multiplied by the radius of the artery, and divided by the wall thickness. Applying the Law of Laplace, ectatic segments of the coronary arteries are frequently under higher intra-arterial pressure, causing further expansion of these vessels [15]. Ultimately, the development of an aneurysm and consequent dissection is associated with the presence of coronary artery ectasia or tortuosity $[16,17]$.

Management of SCAD is greatly disputed since there are no guidelines for therapy or clinical trials; a higher percentage of clinicians favor a conservative approach with therapy akin to treatment after a MI. With conservative therapy the false lumen containing the intramural hematoma will be reabsorbed over time [9]. Revascularization with PCI, CABG, or fibrinolysis remains a treatment option, each performed on a case-bycase base, given the elevated chance of bleeding or dissection propagation. Accepted indications for PCI include left main stem involvement, ongoing symptoms, TIMI 0 flow unlikely to resolve with conservative therapy alone, or hemodynamic instability in the form of sustained ventricular arrhythmias, cardiogenic shock, or evidence of ongoing ischemia $[9,18]$. CABG should be considered when PCI has failed or for left main stem dissections [18]. The proximal segment of the artery

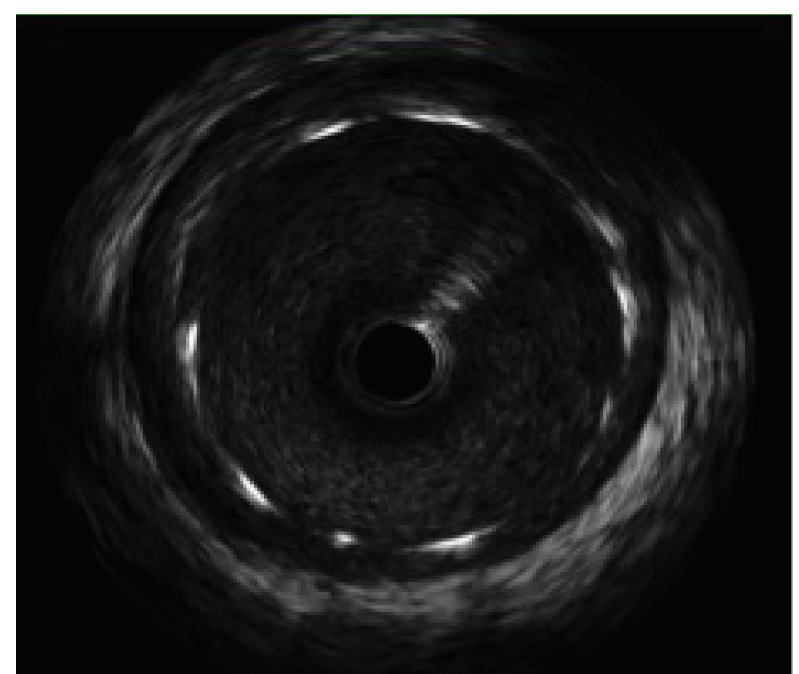

Figure 4. Intravascular ultrasound of the dissection following placement of a drug eluding stent with resolution of the intramural hematoma.

is the ideal location for stenting to limit dissection access and its' associated complications [9]. Conservative therapy is preferred because revascularization with PCI is linked to greater failure rates and complications, which in part is multifactorial [11]. Coronary dissection propagation, inaccurate manipulation with the true and false lumen, increased fragility of the coronary arteries, and complications of stents themselves including stent malposition, thrombosis, and in-stent restenosis contribute to the high failure rate of PCI [19]. Long-term prognosis includes 1- and 10-year mortality and is estimated to be $1.1 \%$ and $7.7 \%$ respectively, with a 10 -year recurrence rate of $30 \%[9]$.

\section{Funding}

No funding was required.

\section{Conflicts of Interest}

All authors of this manuscript have no conflict of interest to disclose.

\section{Abbreviations}

ACS: acute coronary syndrome; CABG: coronary artery bypass grafting; CAD: coronary artery disease; CTD: connective tissue disease; DM: diabetes mellitus; ECHO: echocardiogram; ED: emergency department; EKG: electrocardiogram; FMD: fibromuscular dysplasia; HLD: hyperlipidemia; HTN: hypertension; IBD: inflammatory bowel disease; IVUS: intravascular ultrasound; NSTEMI: non-ST elevation myocardial infarction; MI: myocardial infarction; MM: millimeter; OCT: optical coherence tomography; OM: obtuse marginal branch; 
PCI: percutaneous coronary intervention; RCA: right coronary artery; SCAD: spontaneous coronary artery dissection

\section{References}

1. Puri R, Leong DP, Nicholls SJ, Liew GY, Nelson AJ, Carbone A, Copus B, et al. Coronary artery wall shear stress is associated with endothelial dysfunction and expansive arterial remodelling in patients with coronary artery disease. EuroIntervention. 2015;10(12):1440-1448.

2. Saw J. Spontaneous coronary artery dissection. Can J Cardiol. 2013;29(9):1027-1033.

3. Saw J, Sedlak T. Cardiology patient page. Spontaneous coronary artery dissection (SCAD). Circulation. 2015;131(1):e3-5.

4. Cannon CP. Importance of TIMI 3 flow. 2001. Retrieved November 25, 2017, from http://circ.ahajournals.org/content $/ 104 / 6 / 624$.

5. Lilly LS. Pathophysiology of heart disease: a collaborative project of medical students and faculty. Philadelphia: Wolters Kluwer. 2016.

6. Douglas SP, Saw F, RCPC, FACC J. Coronary Dissection. 2015. Retrieved May 15, 2017, from https://www.uptodate. com/contents/spontaneous-coronary-artery-dissection.

7. Willerson JT. Cardiovascular medicine. London: Springer. 2007.

8. Skelding KA, Hubbard CR. Spontaneous coronary artery dissection related to menstruation. J Invasive Cardiol. 2007;19(6):E174-177.

9. Aziz S. (n.d.). Spontaneous coronary artery dissection. Retrieved August 12, 2017, from https://www.escardio. org/Journals/E-Journal-of-Cardiology-Practice/Volume-14/spontaneous-coronary-artery-dissection.

10. Mieszczanska HZ. Management of cardiovascular disease in women. Place of publication not identified: Springer London Ltd. 2016.

11. Bezerra HG, Costa MA, Guagliumi G, Rollins AM, Simon DI. Intracoronary optical coherence tomography: a comprehensive review clinical and research applications. JACC Cardiovasc Interv. 2009;2(11):1035-1046.

12. Fuster V, Topol EJ, Nabel EG. Atherothrombosis and Coronary Artery Disease. Philadelphia: Wolters Kluwer. 2015.

13. Ectasia. (n.d.). Retrieved November 16, 2017, from http:// medical dictionary.thefreedictionary.com/ectasia.

14. Hartnell GG, Parnell BM, Pridie RB. Coronary artery ectasia. Its prevalence and clinical significance in 4993 patients. Br Heart J. 1985;54(4):392-395.

15. Goz M, Soylemez N, Demirbag R. Images in cardio-thoracic surgery: Multiple spontaneous coronary artery dissection presenting in association with coronary ectasia. Eur J Cardiothorac Surg. 2009;35(5):907.

16. Mavrogeni Sophia. (n.d.). Retrieved November 25, 2017, from https:/www.escardio.org/Journals/E-Journal-ofCardiology-Practice/Volume-8/Coronary-artery-ectasiadiagnosis-and-treatment 907.

17. Hurst JW, Schlant RC, Alexander RW. The Heart, arteries, and veins. New York: McGraw-Hill, Health Professions Div. 1994.

18. Kahles F, Schuh A, Lehrke M, Burgmaier M, Marx $\mathrm{N}$, Reith S. Acute anterior wall infarction due to coronary thrombus due to a spontaneous intimal tear. 2017. Retrieved November 02, 2017, from https:/www. thieme-connect.com/products/ejournals/html/10.1055/s0043-118633\#N65986. http://casereports.bmj.com/content/2017/bcr-2017-222130.long.

19. Vlodaver Z, Wilson RF, Garry DJ. Coronary heart disease: clinical, pathological, imaging, and molecular profiles. Berlin: Springer. 2014. 